\title{
Normative data of olfactory abilities using cultural adaption Sniffin' sticks smell test in different age groups
}

\author{
Salina Husain, MS, ORL-HNS, IrfanAffandiHamid, MD, Farah DayanaZahedi, MS, ORL-HNS, Aneeza Khairiyah Wan Hamizan, PhD.
}

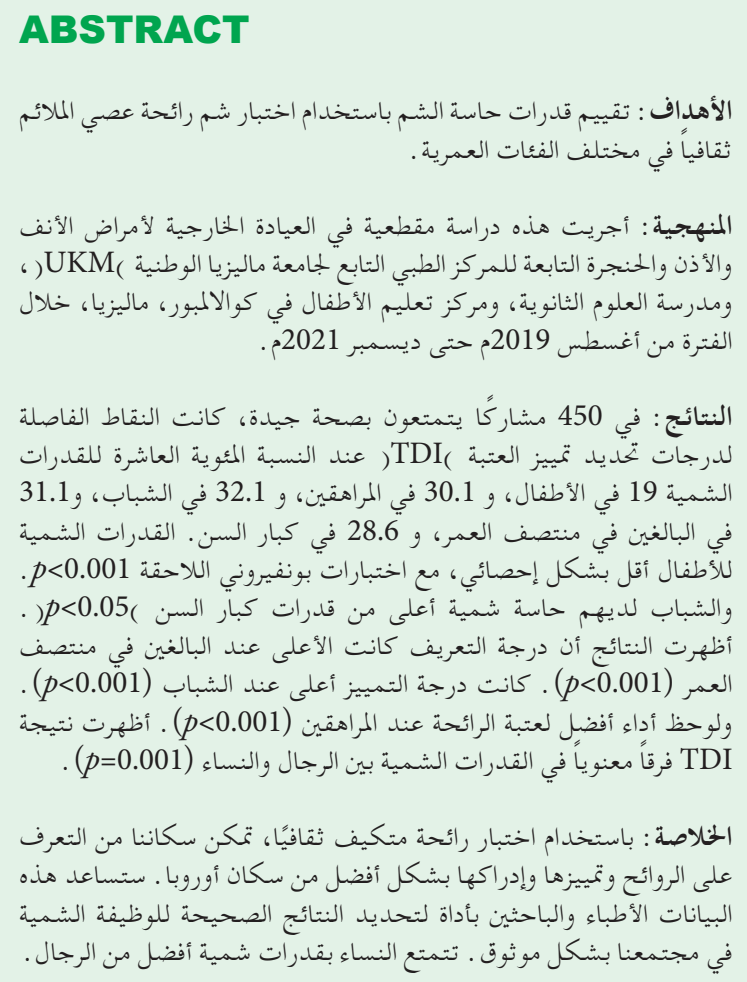

Objectives: To assess olfactory abilities using a culturally adapted Sniffin' Sticks smell test in different age groups.

Methods: This was a cross-sectional study, conducted at Otorhinolaryngology outpatient clinic of Universiti Kebangsaan Malaysia Medical Centre, Secondary Science School, and Children Learning Centre in Kuala Lumpur, Malaysia, from August 2019 until December 2021.

Results: In 450 healthy participants, the cut-off points of the threshold discrimination identification (TDI) scores at the 10th percentile for olfactory abilities were 19 in children, 30.1 in adolescents, 32.1 in young adults, 31.1 in middle-aged adults, and 28.6 in older adults. The children had significantly lower olfactory abilities, with the Bonferroni post-hoc tests of $p<0.001$. The young adults had higher olfactory abilities than older adults $(p<0.05)$. The results showed that the identification score was highest in middle-aged adults $(p<0.001)$. The discrimination score was the highest in young adults $(p<0.001)$. The best odor threshold performance was observed in adolescents $(p<0.001)$. The TDI score showed a significant difference in olfactory abilities between men and women $(p=0.001)$.

Conclusion: Using a culturally adapted smell test, our population was able to identify, discriminate, and perceive odorants better than the European population. This data will help clinicians and researchers with a tool to reliably establish the correct results of olfactory function in our population. Women had better olfactory abilities than men.

Keywords: cultural adaptation, Sniffin' sticks, olfaction, age

Saudi Med J 2021; Vol. 42 (11): 1209-1216 doi: 10.15537/smj.2021.42.11.20210529

From the Department of Otorhinolaryngology-Head and Neck Surgery, Faculty of Medicine, The National University of Malaysia Medical Centre, Kuala Lumpur, Malaysia.

Received 5th July 2021. Accepted 28th September 2021.

Address correspondence and reprint request to: Dr. Salina Husain, Department of Otorhinolaryngology-Head and Neck Surgery, Faculty of Medicine, University Kebangsaan Malaysia Medical Centre, Kuala Lumpur, Malaysia.E-mail: drsalina_h@ukm.edu.my ORCID ID: https://orcid.org/0000-0001-7683-2143

Disclosure. This study was funded by The National University of Malaysia (UKM) Fundamental Research, Kuala Lumpur, Malaysia, grant code of FF-2019-405. 
$\mathrm{O}$ Ifaction, one of the special senses, is the sensation of smell that results from the detection of odors in the environment. The sense of smell determines our ability to perceive a large number of odors that can influence our mood, cognition, and behavior. The sense of smell plays an important role in enhancing the quality of life, and also helps to identify health hazards, such as spoiled food, gas leaks, and fire. ${ }^{1,2}$

In the general population, olfaction disorders have been underestimated as commonly the patients are unaware of their smell disorders. ${ }^{3}$ The prevalence of olfactory disorders is $19 \%$ with $13-16 \%$ accounting for hyposmia, and $5 \%$ for anosmia. ${ }^{4,5}$ It can be categorized as normosmia (normal sense of smell), hyposmia (reduced sense of smell), and anosmia (no sense of smell).

Olfactory dysfunction is common in adults as functions decline with age due to neurodegenerative diseases and cumulative loss of the olfactory epithelium due to repeated infections. ${ }^{1,4,6}$ Olfactory dysfunction in children is rare. Clinical diagnosis of olfactory dysfunction is challenging in children due to low attention span, linguistic development, and lack of odor experience. ${ }^{6}$ Oleszkiewicz et al, ${ }^{7}$ conducted a large database study involving 9139 participants and found women with better olfactory performance than men.

Todate, many psychophysicalandelectrophysiological tests have been developed to quantify olfactory function in clinical settings. ${ }^{8}$ Psychophysical tests are much more popular, as they have more practical and less technical issues than electrophysiological testing. The Sniffin' sticks smell test is a psychophysical test developed by Hummel in 1997 and validated in several European countries. ${ }^{9}$ The test is one of the common olfactory tests used to test nasal chemosensory performance. This semi-objective olfactory test comprises 3 different components testing the sense of smell, the ability to name or identify an odor (odor identification), to differentiate odor (odor discrimination), and to perceive odor at low concentrations (odor thresholds). ${ }^{10}$ The olfactory identification test relies on the participant's familiarity with both the odorant and the descriptors in answering sheets and potentially produces an incorrect diagnosis of hyposmia in a normosmic participant. ${ }^{11,12}$ The threshold discrimination identification (TDI) score is the sum of identification, discrimination, and threshold scores. Normosmia is defined as a TDI score higher than the 10th percentile of the distribution of normal values of the study population according to age and gender. Hyposmia is defined as a TDI score lower than the 10th percentile of the distribution of normal values of the study population according to age and gender. ${ }^{13,14}$ A lower TDI score indicates that the individual has a decreased ability to identify, differentiate, and perceive odors. Evidence showed that different values of TDI scores at the 10th percentile for different age groups. They found that the values were lower in the youngest and oldest age groups.?

Olfactory performance depends on the cultural background. A cultural adaptation of the smell test is recommended in countries with different cultural backgrounds owing to unfamiliar odors and its description or terms used. ${ }^{10,11}$ Hence, a normative data using culture-specific adaptations should be applied to most existing tests to differentiate between normal and abnormal conditions. ${ }^{15}$ The reliable TDI cut-off point for normosmia or hyposmia in different age groups using the cultural adaptation smell test is important to avoid misinterpretation of the test results, leading to a wrong diagnosis. ${ }^{16}$

A validated culturally adapted Malaysian version of the Sniffin' sticks smell test was carried out in a previous study. ${ }^{17}$ However, normative data of olfactory abilities in different age groups in the Malaysian population using this tool needs to be determined to make accurate diagnosis and treatment. The result will help the researcher to establish reliable integrity data of olfaction function locally and permit comparison results obtained from different centers worldwide. ${ }^{12,14}$

Methods. This was a cross-sectional study conducted at 3 centers in Malaysia. The participants were recruited from the Otorhinolaryngology outpatient clinic of Universiti Kebangsaan Malaysia (UKM) Medical Centre, Secondary Science School, and Children Learning Centre in Kuala Lumpur, Malaysia, from August 2019 until December 2021.

A total of 450 healthy participants aged 7-80 years old who passed a culturally adapted Malaysian version of Sniffin' sticks smell identification test (score: $>12$ ) were recruited in the study.

Patients who had immunodeficiency, autoimmune disease, neurogenerative and neuropsychiatric disorders, rhinitis, history of nasal surgery, pregnancy, reduce smell ability, and those with ear, throat, head and neck diseases were excluded. Participants who were recruited from otorhinolaryngology outpatient clinic were normosmic individuals mainly hospital staffs, medical officers, and their relatives. They were given appointments to be seen in the clinic. All participants met the inclusion and exclusion criteria.

Ethical approval for the study was obtained from the Research Ethics Committee of UKMMC (UKM PPI/111/8/JEP-2019-562). This study was performed as per the Declaration of Helsinki for research on human participants. The basic principles such as patient's 
health protection, the knowledge cannot trample rights, followed local regulatory norms, explained risks, burdens and benefits of the study, protection of vulnerable groups and individuals, scientific requirements and research protocols and approval by research ethics committees were granted.

Informed consent was obtained from the participant and the legal guardian for participants under 18 years of age.

The published literature was searched via an electronic database namely PubMed, SCOPUS and EMBASE. The search was performed by using keywords "smell test", "normative data", "sniffin' sticks", "smell test + age", "smell test + olfactory" and "smell test + cultural adaptation". The information provided in the article were counter checked and compared with standard textbooks.

A total of 450 healthy participants were divided into 5 age groups with 90 participants in each group: children ( $7-12$ years), adolescents (13-17 years), young adults (18-40 years), middle-aged adults (41-60 years) and older adults (61-80 years). Demographic data, including the gender of each participant, were recorded.

Participants' olfactory ability was assessed based on identification, discrimination, and threshold using Sniffin' sticks tests (Burghart Messtechnik, Wedel, Germany). The identification test was performed using the culturally adapted Malaysian version of the Sniffin' sticks test. The tip of the pen was placed approximately $2 \mathrm{~cm}$ in front of both nostrils of the participants for approximately 3 seconds. The participants were then asked to identify the odorant from a list of 4 items (including one correct answer and 3 distractors). The odor identification process was repeated using 16 pens with different odors, with a time interval of 20-30 seconds between odor presentations. Participants were asked to choose an option.

For the discrimination and threshold tests, participants were blindfolded using a clean sleeping mask. A set of 16 triplet pens were labeled using green, blue, and red cap numbers from 1-16. The discrimination test demanded the participants' concentration. In the triplet pens, 2 of the pens contained the same odors, and the target pen was discriminated against for having different smells. Using a verbal command by saying the pen's number, the participants were asked to smell the odors. The order presentation of pens varied from triplet-to-triplet set, within 3 seconds of triplet intervals. The participants were asked to choose a number for the target pen. Triplets of pens were presented at intervals of 20-30 seconds.

For the threshold test, the same set of 16 triplet pens concept was used as in the discrimination test. However, the 2 pens contained a blank filled with solvent, and the target pen to be discriminated against had n-butanol. The target pens had different concentrations: set number 16 contained the lowest concentration (highest dilution step) and set number one contained the highest concentration (lowest dilution step). The participants were familiarized with the n-butanol odor using pen number one before beginning the threshold measurements. Participants were tasked to detect which of the 3 pens had n-butanol smell within triplet intervals of 3 seconds and 20-30 seconds between triplets. Starting with the lowest concentration, a staircase paradigm was used until 2 correct answers for the target pen were found marked as the first turning point. After the starting point was detected, the next higher dilution step was offered, and the target pen considered as the second turning point was not correctly identified. The third turning point was where participants correctly identified triplets with higher concentration, twice in a row. The same procedure was performed to obtain the subsequent turning points. The threshold was defined as the mean of the last 4 turning points. Each test had a score ranging from 0-16 points. The TDI score is the sum of the threshold, discrimination, and identification scores. The TDI score was used to assess olfactory abilities.

Statistical analysis. Data was analyzed using the Statistical Package for Social Sciences, Version 26 (IBM Corp., Armonk, NY, USA). The data was descriptively analyzed to get the mean \pm standard deviation and percentile of TDI scores for each age group. The data was explored by examining the statistical significance for the age group to TDI scores at the 10th percentile using analysis of variance (ANOVA). Pairwise comparisons using Bonferroni-corrected multiple comparisons between the 5 age groups. The independent t-test was used to compare TDI score between gender. A 95\% confidence interval was advocated in the analysis. A $p$-value of $<0.05$ was considered significant.

Results. A total of 450 healthy participants were recruited in the study. The mean TDI scores for different age groups were varies. Hyposmia is defined as a TDI score lower than the 10th percentile of the distribution of normal values of the study population according to age and gender. Using a descriptive analysis, the TDI score of more than the 10th percentile is considered as normosmia, the olfactory abilities in different age groups were assessed. We found the cut-off point TDI scores for normosmia in different age groups were varies. The cut-off points of the TDI scores at the 10th percentile for olfactory abilities were 19 in children, 30.1 in adolescents, 32.1 in young adults, 31.1 in 
middle-aged adults, and 28.6 in older adults. The lowest score was observed in children, followed by older adults, while the young adults had the highest score (Table 1). The pairwise comparisons of the TDI scores for all groups using Bonferroni post-hoc tests pointed out that children had significantly lower olfactory ability compared to other groups $(p<0001)$. Young adults showed significantly higher olfactory abilities than older adults $(p<0.05$; Table 2$)$.

We used ANOVA and Bonferroni post-hoc tests to analyse the subsets of the smell test: smell identification, discrimination, and threshold. The results showed that the identification score was highest in middle-aged adults $(p<0.001)$ (Table 3$)$. The discrimination score was the highest in young adults $(p<0.001)$ (Table 4$)$. The best odor threshold performance was observed in adolescents with a mean $p=0.001$. Odor threshold scores declined as the age increased (Table 5).

The TDI score showed a significant difference in olfactory abilities between men and women ( $p=0.001)$ suggesting that women outperformed men throughout the age. There were significant differences between genders in the discrimination $(p<0.001)$ and identification $(p=0.005)$ tests. However, the analysis showed no significant differences in the threshold test $(p=0.478$; Table 6).

Discussion. Smell is critical to avoid environmental and health hazards, such as smoke from the fire, gas leaks and spoiled food. Countries with multiple cultural backgrounds use cultural adaptation smell tests owing to the unfamiliarity of tested odors. Normative data using the cultural adaptation smell test to obtain the cut-off point for hyposmia in different age groups is critical to avoid unreliable test results, leading to an incorrect diagnosis.

A recent study by Oleszkiewicz et al, ${ }^{7}$ showed various cut-off points for normosmia/hyposmia and values at the 10th percentile in the different age groups. They found the cut-off TDI score in the age group of 5-10 years was $19.4,28.5$ in $11-20$ years, 30.8 in $20-30$ years, 30.5 in $31-40$ years, 28.2 in $41-50$ years, 27.3 in $51-60$

Table 1 - Normative values for the culturally adapted Malaysian version of Sniffin sticks smell test.

\begin{tabular}{|c|c|c|c|c|c|c|c|c|c|c|c|c|}
\hline \multirow[t]{2}{*}{ Normosmic } & \multicolumn{4}{|c|}{ Female subjects } & \multicolumn{4}{|c|}{ Male subjects } & \multicolumn{4}{|c|}{ All subjects } \\
\hline & THR & DIS & ID & TDI & THR & DIS & ID & TDI & THR & DIS & ID & TDI \\
\hline \multicolumn{13}{|c|}{ Age group A: children (7-12 years old) } \\
\hline $\mathrm{N}$ & \multicolumn{4}{|c|}{31} & \multicolumn{4}{|c|}{59} & \multicolumn{4}{|c|}{90} \\
\hline Mean & 10.98 & 8.84 & 9.26 & 29.02 & 10.47 & 8.90 & 9.22 & 28.60 & 10.64 & 8.88 & 9.23 & 28.74 \\
\hline $\mathrm{SD}$ & 3.65 & 2.10 & 1.88 & 4.87 & 4.19 & 2.62 & 2.43 & 7.37 & 4.00 & 2.44 & 2.24 & 6.59 \\
\hline Min & 1 & 3 & 5 & 19 & 1 & 3 & 3 & 11 & 1 & 3 & 3 & 11 \\
\hline Max & 16 & 13 & 13 & 37 & 16 & 14 & 13 & 41 & 16 & 14 & 13 & 41 \\
\hline \multicolumn{13}{|l|}{ Percentiles } \\
\hline 5 & 2.20 & 4.80 & 5.60 & 19.60 & 2.00 & 4.00 & 4.00 & 15.00 & 2.00 & 4.55 & 5.00 & 16.55 \\
\hline 10 & 4.60 & 6.20 & 7.00 & 22.60 & 4.00 & 6.00 & 5.00 & 17.00 & 4.05 & 6.00 & 6.10 & 19.00 \\
\hline 25 & 9.00 & 7.00 & 8.00 & 26.00 & 7.50 & 7.00 & 8.00 & 23.00 & 8.00 & 7.00 & 8.00 & 24.00 \\
\hline 50 & 12.00 & 9.00 & 9.00 & 28.50 & 11.00 & 9.00 & 9.00 & 30.00 & 11.75 & 9.00 & 9.00 & 29.75 \\
\hline 75 & 13.00 & 10.00 & 11.00 & 32.50 & 14.00 & 11.00 & 11.00 & 34.00 & 13.63 & 11.00 & 11.00 & 33.50 \\
\hline 90 & 14.90 & 11.80 & 11.00 & 36.00 & 15.00 & 13.00 & 13.00 & 37.00 & 15.00 & 12.00 & 12.00 & 36.95 \\
\hline 95 & 15.40 & 12.40 & 12.40 & 36.40 & 15.00 & 13.00 & 13.00 & 39.00 & 15.00 & 13.00 & 13.00 & 38.45 \\
\hline \multicolumn{13}{|c|}{ Age group B: teenager (13-17 years old) } \\
\hline $\mathrm{N}$ & \multicolumn{4}{|c|}{40} & \multicolumn{4}{|c|}{50} & \multicolumn{4}{|c|}{90} \\
\hline Mean & 13.18 & 11.13 & 10.98 & 35.25 & 13.28 & 10.22 & 10.90 & 34.36 & 13.23 & 10.62 & 10.93 & 34.76 \\
\hline $\mathrm{SD}$ & 1.91 & 2.62 & 1.73 & 4.10 & 1.94 & 2.03 & 1.82 & 3.73 & 1.92 & 2.34 & 1.77 & 3.90 \\
\hline Min & 7 & 6 & 7 & 25 & 8 & 4 & 7 & 25 & 7 & 4 & 7 & 25 \\
\hline $\operatorname{Max}$ & 16 & 16 & 14 & 43 & 16 & 14 & 15 & 45 & 16 & 16 & 15 & 45 \\
\hline \multicolumn{13}{|l|}{ Percentiles } \\
\hline 5 & 10.00 & 6.05 & 8.00 & 28.05 & 9.00 & 7.00 & 8.00 & 27.10 & 9.00 & 7.00 & 8.00 & 28.00 \\
\hline 10 & 10.05 & 7.00 & 8.10 & 29.20 & 10.10 & 7.00 & 9.00 & 30.10 & 10.05 & 7.00 & 9.00 & 30.10 \\
\hline 25 & 12.13 & 9.00 & 10.00 & 32.25 & 12.00 & 9.00 & 9.75 & 32.00 & 12.00 & 9.00 & 10.00 & 32.00 \\
\hline 50 & 13.00 & 12.00 & 11.00 & 35.00 & 14.00 & 10.00 & 11.00 & 35.00 & 14.00 & 11.00 & 11.00 & 35.00 \\
\hline 75 & 14.75 & 13.00 & 12.00 & 39.00 & 15.00 & 12.00 & 12.00 & 36.63 & 15.00 & 12.00 & 12.00 & 37.13 \\
\hline 90 & 15.45 & 14.00 & 13.00 & 40.00 & 15.00 & 13.00 & 13.00 & 39.00 & 15.00 & 13.90 & 13.00 & 40.00 \\
\hline 95 & 15.98 & 15.00 & 14.00 & 42.43 & 16.00 & 13.45 & 14.45 & 40.45 & 16.00 & 14.00 & 14.00 & 41.00 \\
\hline
\end{tabular}

THR: olfactory threshold, DIS: odor discrimination, ID: odor identification, TDI: threshold discrimination identification, SD: standard deviation TDI is a composite score as the sum of results for THR, DIS, and ID by age groups and gender 
Smell abilities in different age groups ... Husain et al

Table 1 - Normative values for the culturally adapted Malaysian version of Sniffin Sticks Smell Test (continued).

\begin{tabular}{|c|c|c|c|c|c|c|c|c|c|c|c|c|}
\hline \multirow[t]{2}{*}{ Normosmic } & \multicolumn{4}{|c|}{ Female subjects } & \multicolumn{4}{|c|}{ Male subjects } & \multicolumn{4}{|c|}{ All subjects } \\
\hline & THR & DIS & ID & TDI & THR & DIS & ID & TDI & THR & DIS & ID & TDI \\
\hline \multicolumn{13}{|c|}{ Age group C: young adult (18-40 years old) } \\
\hline $\mathrm{N}$ & \multicolumn{4}{|c|}{60} & \multicolumn{4}{|c|}{30} & \multicolumn{4}{|c|}{90} \\
\hline Mean & 11.54 & 12.23 & 12.80 & 36.38 & 11.54 & 12.23 & 12.80 & 36.38 & 11.58 & 12.08 & 12.73 & 36.26 \\
\hline SD & 1.89 & 1.45 & 1.41 & 2.87 & 1.89 & 1.45 & 1.41 & 2.87 & 2.07 & 1.55 & 1.50 & 2.96 \\
\hline Min & 7.25 & 9 & 9 & 29.5 & 7.25 & 9 & 9 & 29.50 & 7 & 7 & 9 & 29.50 \\
\hline Max & 15.50 & 16 & 16 & 41.50 & 15.50 & 16 & 16 & 41.50 & 15.50 & 16 & 16 & 43.50 \\
\hline \multicolumn{13}{|l|}{ Percentiles } \\
\hline 5 & 8.00 & 9.05 & 10.05 & 31.26 & 8.00 & 9.05 & 10.05 & 31.26 & 7.89 & 9.00 & 10.00 & 31.14 \\
\hline 10 & 8.55 & 10.10 & 11.00 & 32.50 & 8.55 & 10.10 & 11.00 & 32.50 & 8.05 & 10.00 & 11.00 & 32.05 \\
\hline 25 & 10.50 & 11.00 & 12.00 & 34.50 & 10.50 & 11.00 & 12.00 & 34.50 & 10.50 & 11.00 & 12.00 & 34.50 \\
\hline 50 & 11.50 & 12.00 & 13.00 & 36.38 & 11.50 & 12.00 & 13.00 & 36.38 & 11.50 & 12.00 & 13.00 & 35.75 \\
\hline 75 & 12.50 & 13.00 & 14.00 & 39.00 & 12.50 & 13.00 & 14.00 & 39.00 & 13.50 & 13.00 & 14.00 & 38.50 \\
\hline 90 & 14.45 & 14.00 & 15.00 & 40.50 & 14.45 & 14.00 & 15.00 & 40.50 & 14.50 & 14.00 & 15.00 & 40.50 \\
\hline 95 & 14.50 & 14.00 & 15.00 & 41.23 & 14.50 & 14.00 & 15.00 & 41.23 & 14.73 & 14.00 & 15.00 & 41.36 \\
\hline \multicolumn{13}{|c|}{ Age group D: middle age adult (41-60 years old) } \\
\hline $\mathrm{N}$ & \multicolumn{4}{|c|}{54} & \multicolumn{4}{|c|}{36} & \multicolumn{4}{|c|}{90} \\
\hline Mean & 11.41 & 11.85 & 13.06 & 36.26 & 11.42 & 11.72 & 12.83 & 35.81 & 11.41 & 11.80 & 12.97 & 36.08 \\
\hline $\mathrm{SD}$ & 2.48 & 1.39 & 1.31 & 3.54 & 2.30 & 1.16 & 1.70 & 3.28 & 2.40 & 1.30 & 1.47 & 3.43 \\
\hline Min & 5 & 8 & 10 & 25.50 & 3.50 & 10 & 8 & 30 & 3.50 & 8 & 8 & 25.50 \\
\hline Max & 15.50 & 15 & 16 & 42 & 14.50 & 14 & 16 & 43 & 15.50 & 15 & 16 & 43 \\
\hline \multicolumn{13}{|l|}{ Percentiles } \\
\hline 5 & 6.25 & 9.00 & 11.00 & 29.50 & 7.33 & 10.00 & 8.85 & 30.43 & 6.50 & 9.55 & 11.00 & 30.28 \\
\hline 10 & 7.25 & 10.00 & 11.00 & 30.75 & 8.35 & 10.00 & 11.00 & 31.35 & 8.00 & 10.00 & 11.00 & 31.05 \\
\hline 25 & 10.38 & 11.00 & 12.00 & 34.38 & 10.00 & 11.00 & 12.00 & 33.13 & 10.00 & 11.00 & 12.00 & 33.88 \\
\hline 50 & 12.00 & 12.00 & 13.00 & 37.00 & 11.63 & 12.00 & 13.00 & 35.25 & 11.88 & 12.00 & 13.00 & 36.50 \\
\hline 75 & 13.00 & 13.00 & 14.00 & 39.00 & 13.00 & 13.00 & 14.00 & 38.38 & 13.00 & 13.00 & 14.00 & 38.50 \\
\hline 90 & 14.50 & 13.00 & 15.00 & 40.50 & 14.00 & 13.00 & 14.30 & 40.30 & 14.00 & 13.00 & 14.90 & 40.00 \\
\hline 95 & 15.5 & 13.25 & 15.25 & 41.13 & 14.08 & 13.15 & 16.00 & 42.15 & 14.50 & 13.00 & 15.45 & 41.23 \\
\hline \multicolumn{13}{|c|}{ Age group E: older adults (61-80 years old) } \\
\hline $\mathrm{N}$ & \multicolumn{4}{|c|}{40} & \multicolumn{4}{|c|}{50} & \multicolumn{4}{|c|}{90} \\
\hline Mean & 10.78 & 11.18 & 12.60 & 34.48 & 10.45 & 11.18 & 12.86 & 34.17 & 10.59 & 11.18 & 12.74 & 34.31 \\
\hline SD & 2.08 & 1.28 & 1.22 & 3.41 & 2.10 & 1.27 & 1.46 & 4 & 2.08 & 1.27 & 1.35 & 3.73 \\
\hline Min & 5 & 8 & 10 & 26 & 4 & 8 & 10 & 24 & 4 & 8 & 10 & 24 \\
\hline Max & 16 & 14 & 15 & 40 & 13 & 13 & 16 & 40 & 16 & 14 & 16 & 40 \\
\hline \multicolumn{13}{|l|}{ Percentiles } \\
\hline 5 & 7.53 & 8.05 & 10.05 & 28.00 & 5.88 & 8.55 & 10.55 & 27.10 & 7.28 & 8.55 & 10.55 & 28.00 \\
\hline 10 & 8.00 & 10.00 & 11.00 & 29.20 & 7.50 & 10.00 & 11.00 & 28.50 & 8.00 & 10.00 & 11.00 & 28.55 \\
\hline 25 & 9.50 & 10.00 & 12.00 & 32.63 & 9.50 & 10.00 & 12.00 & 31.50 & 9.50 & 10.00 & 12.00 & 31.88 \\
\hline 50 & 11.00 & 11.00 & 13.00 & 34.50 & 11.00 & 11.50 & 13.00 & 34.00 & 11.00 & 11.00 & 13.00 & 34.00 \\
\hline 75 & 12.00 & 12.00 & 13.75 & 37.00 & 12.00 & 12.00 & 14.00 & 38.00 & 12.00 & 12.00 & 14.00 & 37.13 \\
\hline 90 & 13.00 & 12.90 & 14.00 & 39.00 & 12.95 & 13.00 & 14.90 & 39.00 & 13.00 & 13.00 & 14.00 & 39.00 \\
\hline 95 & 13.48 & 13.00 & 14.00 & 39.95 & 13.00 & 13.00 & 16.00 & 40.00 & 13.00 & 13.00 & 15.00 & 40.00 \\
\hline
\end{tabular}

THR: olfactory threshold, DIS: odor discrimination, ID: odor identification, TDI: threshold discrimination identification, SD: standard deviation, TDI is a composite score as the sum of results for THR, DIS, and ID by age groups and gender

Table 2 - The differences between mean TDI scores of 2 age groups.

\begin{tabular}{lcccc}
\hline Age group & Children $(17-12$ years $)$ & Adolescents $(13-17$ years $)$ & Young adults $(18-40$ years $)$ & Middle-aged adults $(41-60$ years $)$ \\
\hline Adolescents (13-17 years) & $-6.011(p<0.001)$ & & & \\
Young adults (18-40 years) & $-7.517(p<0.001)$ & $-1.506(p=0.197)$ & & \\
Middle-aged adults (41-60 years) & $-7.339(p<0.001)$ & $-1.328(p=0.396)$ & $0.178(p=1.000)$ & $1.956(p<0.050)$ \\
Older adults (61-80 years) & $-5.561(p<0.001)$ & $0.450(p=1.000)$ & $1.778(p=0.060)$ \\
\hline
\end{tabular}

The differences between mean scores of 2 age groups (group in a column - group in a row) and the post-hoc test: Bonferroni correction significance level $(p<0.001 ; p<0.05)$ 
Smell abilities in different age groups ... Husain et al

Table 3 - The differences between mean smell sub-test (identification test) scores of 2 age groups.

\begin{tabular}{|c|c|c|c|c|}
\hline Age group & Children ( $7-12$ years) & Adolescents (13-17 years) & Young adults (18-40 years) & Middle-aged adults ( $41-60$ years) \\
\hline Adolescents (13-17 years) & $-1.698(p<0.001)$ & & & \\
\hline Young adults ( $18-40$ years) & $-3.509(p<0.001)$ & $-1.810(p<0.001)$ & & \\
\hline Middle-aged adults ( $41-60$ years) & $-3.742(p<0.001)$ & $-2.044(p<0.001)$ & $-0.233(p=0.359)$ & \\
\hline Older adults (61-80 years) & $-3.520(p<0.001)$ & $-1.821(p<0.001)$ & $-0.011(p=0.965)$ & $-0.222(p=0.383)$ \\
\hline
\end{tabular}

Table 4 - The differences between mean smell subtest (discrimination test) scores of 2 age groups.

\begin{tabular}{lcccc}
\hline Age group & Children $(7-12$ years $)$ & Adolescents $(13-17$ years $)$ & Young adults $(18-40$ years $)$ & Middle-aged adults $(41-60$ years $)$ \\
\hline Adolescents (13-17 years) & $-1.772(p<0.001)$ & & & \\
Young adults (18-40 years) & $-3.224(p<0.001)$ & $-1.451(p<0.001)$ & $0.278(p=0.313)$ & $0.622(p<0.05)$ \\
Middle-aged adults (41-60 years) & $-2.946(p<0.001)$ & $-1.174(p<0.001)$ & $0.900(p<0.05)$ & $0.551(p<0.05)$ \\
Older adults (61-80 years) & $-2.324(p<0.001)$ & $-0.55)$ & \\
\hline
\end{tabular}

The differences between mean scores of 2 age groups (group in a column - group in a row) and the post-hoc test: Bonferroni correction significance level $(p<0.001 ; p<0.05)$

Table 5 - The differences between mean smell subtest (threshold test) scores of 2 age groups.

\begin{tabular}{|c|c|c|c|c|}
\hline Age group & Children ( $7-12$ years) & Adolescents (13-17 years) & Middle-aged adults (41-60 years) & Older adults (61-80 years) \\
\hline Adolescents (13-17 years) & $-2.590(\mathrm{p}<0.001)$ & & & \\
\hline Young adults (18-40 years) & $-0.954(\mathrm{p}<0.05)$ & $1.636(\mathrm{p}<0.001)$ & & \\
\hline Middle-aged adults (41-60 years) & $-0.785(\mathrm{p}<0.05)$ & $1.806(\mathrm{p}<0.001)$ & $0.169(\mathrm{p}=0.665)$ & \\
\hline Older adults (61-80 years) & $0.035(\mathrm{p}=0.929)$ & $2.625(\mathrm{p}<0.001)$ & $0.989(\mathrm{p}<0.05)$ & $0.819(\mathrm{p}<0.05)$ \\
\hline
\end{tabular}

The differences between mean scores of 2 age groups (group in a column - group in a row) and the post-hoc test: Bonferroni correction significance level $(p<0.001 ; p<0.05)$

Table 6 - The differences of mean TDI and subtests scores between gender using paired T-test .

\begin{tabular}{|c|c|c|c|}
\hline \multirow[t]{2}{*}{ Variable } & \multicolumn{2}{|c|}{ Scores } & \multirow[t]{2}{*}{$P$-value } \\
\hline & $\operatorname{Mean} \pm S D$ & $\begin{array}{l}\text { Mean difference } \\
\quad(95 \% \mathrm{CI})\end{array}$ & \\
\hline \multicolumn{4}{|l|}{$T H R$} \\
\hline $\begin{array}{l}\text { Female } \\
\text { Male }\end{array}$ & $\begin{array}{l}11.59 \pm 2.49 \\
11.40 \pm 3.03\end{array}$ & $\begin{array}{c}0.19(-0.33- \\
0.70)\end{array}$ & 0.478 \\
\hline \multicolumn{4}{|l|}{$D I S$} \\
\hline $\begin{array}{l}\text { Female } \\
\text { Male }\end{array}$ & $\begin{array}{l}11.29 \pm 2.06 \\
10.53 \pm 2.21\end{array}$ & $0.76(0.36-1.15)$ & $<0.001$ \\
\hline \multicolumn{4}{|l|}{$I D$} \\
\hline $\begin{array}{l}\text { Female } \\
\text { Male }\end{array}$ & $\begin{array}{l}12.01 \pm 1.98 \\
11.43 \pm 2.42\end{array}$ & $0.21(0.17-0.99)$ & 0.005 \\
\hline \multicolumn{4}{|l|}{$T D I$} \\
\hline $\begin{array}{l}\text { Female } \\
\text { Male } \\
\end{array}$ & $\begin{array}{l}34.80 \pm 4.37 \\
33.26 \pm 5.64\end{array}$ & $1.53(0.60-2.47)$ & 0.001 \\
\hline \multicolumn{4}{|c|}{$\begin{array}{l}\text { TDI: threshold discrimination identification, SD: standard deviation, CI } \\
\text { confidence interval, THR: olfactory threshold, DIS: odor discrimination, } \\
\text { ID: odor identification. Threshold discrimination identification is a } \\
\text { composite score as the sum of results for THR, DIS and ID }\end{array}$} \\
\hline
\end{tabular}

years, 24.9 in $61-70$ years, 19.2 in $71-80$ years, and 13 in $>80$ years. The age group more than 80 years had the lowest score: age group 71-80 years was the second lowest, followed by the age group 5-10 years. ${ }^{7}$

In our study, we found a similar result pattern for the 10th percentile TDI score in different age groups. Our study revealed the cut-off TDI score was 19 for patients aged 7-12 years, 30.1 for the age group 13-17 years, 32.1 for the age group 18-40 years, 31.1 for the age group 41-60 years, and 28.6 for the age group 61-80 years. However, we found that the cut-off points of the TDI score for normosmia/hyposmia in adolescents, young adults, middle-aged and older adults were higher in our population than that in the European population. Furthermore, our study found that the lowest 10th percentile TDI score was observed in children, followed by older adults.

Kobal et $a l,{ }^{13}$ found that the age group 6-15 years had a better score on the identification test compared to other 
groups. On the contrary, Oleszkiewicz et al, ${ }^{7}$ found that the age group 31-40 years had a significant increase in identification test scores. Our study revealed a different result whereby the middle-aged adults (41-60 years) had significantly higher scores on the identification test. It means that middle-aged population can identify odors better than other age groups.

Kobal et al, ${ }^{13}$ found that the 16-35 years age group had a higher discrimination score than $>55$ years group. Oleszkiewicz et al, ${ }^{7}$ also found the highest discrimination score was in the age group 21-30 years. Our study showed similar result that young adults (19-40 years) had better discrimination scores compared to other age groups $(p<0.05)$, suggesting that young adults had the highest ability to differentiate or discriminate odors.

Kobal et al, ${ }^{13}$ revealed the age group 6-15 years had a significantly higher smell threshold than the 16-55 years group, and the 16-35 years age group had a better threshold than the $>55$ years group. Oleszkiewicz et $\mathrm{al},{ }^{7}$ found the highest threshold score in the age group of 21-30 years. Our study was similar to Kobal et al. ${ }^{13}$ We found the best odor threshold performance was seen in adolescents (13-18 years), this means that the adolescents had the highest ability to perceive odors. Olfactory ability declined as the age increased. A study by Delgado-Losada et al, ${ }^{9}$ showed a significant decrease in odor thresholds with age. Oleszkiewicz et $\mathrm{al}^{7}{ }^{7}$ also reported the most pronounced loss with age in the odor thresholds test. Our study showed a similar result: the olfactory threshold declined with age. This supports the idea that it may cause damage to the olfactory system and neurodegenerative disease-causing olfactory threshold performance to decline with age.

A study involving a large sample size of 9139 participants showed a significant difference in olfactory performance between genders where women outperformed men $(p<0.001) .^{7}$ Our study also found that women had significantly better olfactory abilities compared to men with a $p=0.001$. Women in our population had a significantly higher ability to identify and discriminate odors.

Study limitations. A long duration was required to complete all the tests, causing a loss in participants' attention span. Difficulties in following instructions during the procedure in some of the participants may be due to the complexities of the test or the language barrier.

Our study found that the cut-off points for olfactory abilities using culturally adapted Malaysian version of sniffin' sticks smell test in our population was higher than the European population. Therefore, the cut-off points of TDI scores by Western studies can't be implemented in our population. The cut-off scores of normosmia/hyposmia is crucial to establish the correct results of olfactory function in our population. It will be used by clinicians and in all future smell research in Malaysian population.

In conclusion, using a culturally adapted smell test, our population was able to identify, discriminate, and perceive odorants better than the European population. This study will help clinicians and researchers with a tool to reliably establish the correct results of olfactory function in our population.

Acknowledgment. The authors gratefully acknowledge the volunteers for their participation in the study. We also acknowledge the help of doctors and staffs of the Otorhinolaryngology Department, Secondary Science School, and Children Learning Centre, Kuala Lumpur, Malaysia. We would like to thank Editage (www.editage.com) for English language editing.

\section{References}

1. Dong Y, Wang Y, Liu K, Liu R, Tang S, Zhang Q, et al. Olfactory impairment among rural-dwelling Chinese older adults: prevalence and associations with demographic, lifestyle, and clinical factors. Front Aging Neurosci 2021; 13: 621619.

2. Attems J, Walker L, Jellinger KA. Olfaction and aging: a minireview. Gerontology 2015; 61: 485-490.

3. Nguyen DT, Rumeau C, Gallet P, Jankowski R. Olfactory exploration: state of the art. Eur Ann Otorbinolaryngol Head Neck Dis 2016; 133: 113-118.

4. Pinkaew B, Assanasen P, Bunnag C. Smell discrimination and identification scores in Thai adults with normosmia. Asian Biomedicine 2015; 9: 789-795.

5. Liu G, Zong G, Doty RL, Sun Q. Prevalence and risk factors of taste and smell impairment in a nationwide representative sample of the US population: a cross-sectional study. $B M J$ Open 2016; 6: e013246.

6. Gellrich J, Stetzler C, Oleszkiewicz A, Hummel T, Schriever VA. Olfactory threshold and odor discrimination ability in children - evaluation of a modified "sniffin' sticks" test. Sci Rep 2017; 7: 1928.

7. Oleszkiewicz A, Schriever VA, Croy I, Hähner A, Hummel T. Updated sniffin' sticks normative data based on an extended sample of 9139 subjects. Eur Arch Otorhinolaryngol 2019; 276: 719-728.

8. Su B, Bleier B, Wei Y, Wu D. Clinical implications of psychophysical olfactory testing: assessment, diagnosis, and treatment outcome. Front Neurosci 2021; 15: 646956.

9. Delgado-Losada ML, Delgado-Lima AH, Bouhaben J. Spanish validation for olfactory function testing using the sniffin' sticks olfactory test: threshold, discrimination, and identification. Brain Sci 2020; 10: 943.

10. Tekeli H, Altundağ A, Salihoğlu M, Cayönü M, Kendirli MT. The applicability of the "sniffin' sticks" olfactory test in a Turkish population. Med Sci Monit 2013; 19: 1221-1226.

11. Reddy G, Zak JD, Vergassola M, Murthy VN. Antagonism in olfactory receptor neurons and its implications for the perception of odor mixtures. Elife 2018; 7: e34958. 
12. Čičelienė J, Vaičys Ž, Rastenytė D. Development of the lithuanian version of sniffin' sticks 12 odor identification test. Medicina (Kaunas) 2018; 54: 13.

13. Kobal G, Klimek L, Wolfensberger M, Gudziol H, Temmel A, Owen CM, et al. Multicenter investigation of 1,036 subjects using a standardized method for the assessment of olfactory function combining tests of odor identification, odor discrimination, and olfactory thresholds. Eur Arch Otorhinolaryngol 2000; 257: 205-211.

14. Rumeau C, Nguyen DT, Jankowski R. How to assess olfactory performance with the sniffin' sticks test $\left({ }^{\circ}\right)$. Eur Ann Otorhinolaryngol Head Neck Dis 2016; 133: 203-206.
15. Ribeiro JC, Simóes J, Silva F, Silva ED, Hummel C, Hummel $\mathrm{T}$, et al. Cultural adaptation of the Portuguese version of the "sniffin' sticks" smell test: reliability, validity, and normative data. PLoS One 2016; 11: e0148937.

16. Fornazieri MA, dos Santos CA, Bezerra TF, Pinna Fde R, Voegels RL, Doty RL. Development of normative data for the Brazilian adaptation of the university of Pennsylvania smell identification test. Chem Senses 2015; 40: 141-149.

17. Sai-Guan L, Husain S, Zahedi FD, Ahmad N, Gendeh BS. Cultural adaptation of sniffin' sticks smell identification test: the Malaysian version. Iran J Otorhinolaryngol 2020; 32: 213-222. 